\title{
Walking the Fine Line of Axillary Management in Mastectomy Patients
}

\author{
Carla Suzanne Fisher, MD \\ Indiana University School of Medicine, Indianapolis
}

While breast-conservation surgery (BCS) represented tremendous progress for breast cancer patients in the 1990s, the twenty first century has been significant for advancements in how we manage the axilla for these same patients. Axillary lymph node dissection (ALND) was previously the standard of care for the management of node-positive disease, but carefully selected patients may now undergo sentinel lymph node biopsy (SLNB) alone ${ }^{1-3}$ or axillary radiation, ${ }^{4,5}$ with oncologically equivalent results and less morbidity. In contrast to the de-escalation of axillary surgery, the role of postmastectomy radiation therapy (PMRT) has increased. ${ }^{6}$ As a result, the management of the axilla and use of PMRT in node-positive patients undergoing mastectomy is complex and variable. In this issue of Annals of Surgical Oncology, the inconsistency in how we manage these patients is highlighted.

Gaines et al. utilized the National Cancer Data Base (NCDB) to analyze patterns of axillary management and PMRT in clinically negative but pathologically node-positive patients undergoing mastectomy between 2013 and 2014. This did not include patients who underwent neoadjuvant chemotherapy. Their goal was to examine how nodal status affected the type of axillary surgery and how extent of surgery interacted with PMRT. Based on this data (from 2013 to 2014), expected management would include SLNB followed by ALND, with the possible exception of those patients with micrometastatic disease. Of the 8089 patients identified in the study, $23.3 \%$ of the cohort had micrometastatic disease and $76.7 \%$ had macrometastatic disease. When the type of axillary procedure performed

(C) Society of Surgical Oncology 2018

First Received: 20 March 2018;

Published Online: 24 April 2018

C. S. Fisher, MD

e-mail: fishercs@iu.edu was analyzed, 52.7\% of patients underwent traditional management of the clinically node-negative axilla, and underwent an SLNB followed by an ALND. However, $30.7 \%$ of these patients underwent SLNB alone and $16.6 \%$ proceeded straight to ALND. Not surprisingly, compared with patients with macrometastatic disease, patients with micrometastatic disease were more likely to undergo SLNB alone, and ALND was more likely if more than one node was positive.

The group also analyzed the use of PMRT and how it may relate to the type of axillary surgical procedure performed. They report that, in this cohort of one to three node-positive patients, $37.3 \%$ underwent PMRT. As expected, with increasing node positivity, there is increased use of PMRT. Furthermore, the authors note several factors associated with receipt of PMRT, including the type of axillary procedure. Using multivariable, binary logistic regression analyses, they demonstrate a significant relationship between receipt of PMRT and use of SLNB alone, suggesting that as we choose to omit ALND, we may be increasing the use of PMRT.

This group previously studied the interaction between axillary surgery and PMRT. ${ }^{7}$ Again, using the NCDB, they demonstrated that the proportion of mastectomy patients undergoing SLNB alone and PMRT increased from 5.2\% in 1998 to $23.8 \%$ in 2011. There was also an overall increase in the use of PMRT in a similar patient population, from $17.5 \%$ in 1998 to $24.1 \%$ in 2009 , to $28.3 \%$ in 2011 . Based on the numbers from the current analysis, these trends continue to increase.

There are a wealth of data points in this current analysis of the NCDB of node-positive mastectomy patients, with two overarching themes: a trend away from ALND, with increased use of PMRT, but significant overall variability in management. The AMAROS and OTOASOR trials demonstrated the oncological equivalence of ALND and radiation therapy, although publication of this data would 
not necessarily have overlapped with the time period of data acquisition reported by Gaines et al. ${ }^{4,5}$ Based on this, in patients who require PMRT, the use of axillary radiation and omission of ALND seems reasonable and is supported by clinical data. Many mastectomy patients also undergo breast reconstruction, and the use of PMRT can affect the utilization, timing, and quality of breast reconstruction. While the rates of reconstruction do not appear to be affected in similar patient populations, it is an important factor that is missing in this particular analysis. ${ }^{6}$ If we omit ALND but replace with PMRT, what are the consequences for breast reconstruction?

Regarding variability, in $19 \%$ of patients SLNB was the only treatment for their node-positive disease, yet $22.6 \%$ of patients with micrometastatic disease underwent PMRT. Controversy remains for the use of PMRT for one to three positive lymph nodes, and this likely accounts for some of the ongoing inconsistency we see in these patients. We eagerly await the publication of the SUPREMO trial (Selective Use of Postoperative Radiation aftEr MastectOmy), which will address the benefit, or lack of benefit, of radiation following mastectomy for one to three positive nodes. ${ }^{8}$ Patients in SUPREMO are mandated to undergo ALND, therefore it will not specifically address surgical axillary management but will provide answers regarding PMRT, and subsequently inform the surgical management, in this nebulous patient population.

This large, retrospective database enables us to evaluate modern practice patterns, as well as factors that appear to increase the likelihood of different types of axillary management and PMRT. As healthcare providers, we do not appear to be consistent in our patterns of treating patients with node-positive disease undergoing mastectomy. In a recent editorial, Morrow addresses this with a succinct review of options for the node-positive axilla. ${ }^{9}$ While there is undoubtedly variability nationally, perhaps there is consistency within each health system-again, not something we can determine from this database but something that we should strive for in our own practices.

There is hope for better data regarding best practice in these patients. The UK multicenter, non-inferiority trial POSNOC (POsitive Sentinel NOde: Adjuvant Therapy Alone Versus Adjuvant Therapy Plus Clearance or Axillary Radiotherapy) randomized early-stage breast cancer patients with one to two positive nodes to axillary treatment versus no axillary treatment. ${ }^{10}$ Moreover, both mastectomy and BCS patients are included and this will be stratified.

As surgeons managing patients with breast cancer, we have seen significant advancements in patient care, with better outcomes. While we continue to provide personalized care for each patient, we must strive to be consistent, and evidence-based, in our management.

\section{REFERENCES}

1. Giuliano AE, Hunt KK, Ballman KV, Eitsch PD, Whitworth PW, Leitch AM, et al. Axillary vs no axillary dissection in women with invasive breast cancer and sentinel node metastasis: a randomized clinical trial. JAMA 2011; 305:569-75.

2. Galimberti V, Cole BF, Zurrida S, et al. Axillary dissection versus no axillary dissection in patients with sentinel-node micrometastases (IBCSG 23-01): a phase 3 randomised controlled trial. Lancet Oncol. 2013; 14:297-305.

3. Sola M, Alberro JA, Fraile M, et al. Complete axillary lymph node dissection versus clinical follow-up in breast cancer patients with sentinel node micrometastasis: final results from the multicenter clinical trial AATRM 048/13/2000. Ann Surg Oncol. 2013; 20:120-7.

4. Donker M, Van Tienhoven G, Staver ME, et al. Radiotherapy or surgery of the axilla after a positive sentinel node in breast cancer (EORTC 10981-22023 AMAROS): a randomized, multicenter, open-label, phase 3 non-inferiority trial. Lancet Oncol. 2014; 15:1303-10.

5. Savolt A, Peley G, Polgar C, et al. Eight-year follow up result of the OTOASOR trial: the Optimal Treatment of the Axilla-Surgery or Radiotherapy after positive sentinel lymph node biopsy in early-stage breast cancer: a randomized, single centre, phase III, non-inferiority trial. Eur J Surg Oncol. 2017; 43:672-9.

6. Frasier LL, Holden S, Holden T, et al. Temporal trends in postmastectomy radiation therapy and breast reconstruction associated with changes in National Comprehensive Cancer Network guidelines. JAMA Oncol. 2016; 2:95-101.

7. Yao K, Liederbach E, Pesce C, Wang C, Winchester D. Impact of the American College of Surgeons Oncology Group Z0011 randomized trial on the number of axillary nodes removed for patients with early-stage breast cancer. J Am Coll Surg. 2015; 221:71-81.

8. Thomas JS, Hanby AM, Russell N, et al. The BIG 2.04 MRC/ EORTC SUPREMO Trial: pathology quality assurance of a large phase 3 randomised international clinical trial of postmastectomy radiotherapy in intermediate-risk breast cancer. Breast Cancer Res Treat. 2017;163:63-9.

9. Morrow M. Management of the node-positive axilla in breast cancer in 2017: selecting the right option. JAMA Oncol. 2018; $4: 250-1$.

10. Goyal A, Dodwell D. POSNOC: a randomised trial looking at axillary treatment in women with one or two sentinel nodes with macrometastases. Clin Oncol. 2015; 27:692-5. 